\title{
Protective Effect of Lycopene against Reperfusion Injury in Rats with Ovarian Torsion: A Biochemical and Histopathological Evaluation
}

\author{
Yunus Emre Topdagi ${ }^{5}$ Zekai Halici ${ }^{6}$ \\ ${ }^{1}$ Department of Gynecology and Obstetrics, Faculty of Medicine, \\ Ataturk University, Erzurum, Turkey \\ 2Department of Biochemistry, Faculty of Pharmacy, Ağrı Ibrahim \\ Cecen University, Agri, Turkey \\ ${ }^{3}$ Department of Pathology, Faculty of Medicine, Ataturk University, \\ Erzurum, Turkey \\ ${ }^{4}$ Department of Biochemistry, Faculty of Medicine, Ataturk \\ University, Erzurum, Turkey \\ ${ }^{5}$ Department of Gynecology and Obstetrics, Faculty of Medicine, \\ Sanko University, Gaziantep, Turkey \\ ${ }^{6}$ Department of Pharmacology, Faculty of Medicine, Ataturk \\ University, Erzurum, Turkey
}

Emsal Pinar Topdagi Yilmaz ${ }^{1}$ Harun Un² Betul Gundogdu ${ }^{3} \quad$ Elif Polat ${ }^{4}$ Seda Askin ${ }^{4}$

J Lab Physicians:2020;12:32-37

\begin{abstract}
Address for correspondence Yunus Emre Topdagi, MD, Department of Gynecology and Obstetrics, Faculty of Medicine, Sanko University, Gazimuhtar Paşa Bulvarı No, 36, Şehitkamil Gaziantep 27090, Turkey (e-mail: emretopdagi@hotmail.com).
\end{abstract}

\begin{abstract}
Keywords

- ischemia/reperfusion

- lycopene

- ovarian torsion

- oxidative stress
\end{abstract}

Objective The aim of our study was to evaluate the effect of two different doses of lycopene, an antioxidant, on experimentally induced ovarian ischemia/reperfusion (IR) injury in rat model.

Materials and Methods Twenty-four female rats were randomly divided into four groups: sham operation (group 1), 3-hour ischemia, 3-hour reperfusion (IR) (group 2), and IR $+100 \mathrm{mg} / \mathrm{kg}$ lycopene (PO) (group 3), IR + $200 \mathrm{mg} / \mathrm{kg}$ of lycopene (group 4). The rats' superoxide dismutase (SOD), myeloperoxidase (MPO) activities, malondialdehyde (MDA), and glutathione (GSH) levels were calculated. Ovarian tissue damage was assessed using a histopathological scoring system.

Results Serum parameter levels and histological scores showed that treatment with lycopene may be conservative approach to prevent IR injury after the ovarian detorsion procedure. The improvement with lycopene was higher at $200 \mathrm{mg}$ than at $100 \mathrm{mg}$. The MPO and MDA values were significantly lower in groups 3 and 4 as compared with group $2(p<0.05)$, whereas the MPO and MDA values were lower in group 4 as compared with group 3.The SOD and GSH values were significantly higher in groups 3 and 4 as compared with group $2(p<0.05)$, whereas the SOD and GSH values were higher in group 4 as compared with group 3. Tissue damage scores were elevated in the IR group compared with the sham group, but the treatment with different lycopene doses after reperfusion improved the histopathological tissue damage scores.

Conclusion The results showed that lycopene treatment reduced ovarian IR damage. Antioxidant activity was found to increase in a dose-dependent manner. Lycopene treatment may be conservative approach for ovarian torsion patients after the detorsion procedure to prevent IR damage.
License terms

() (1) $\ominus \circledast$ 


\section{Introduction}

Ovarian torsion is a gynecological emergency among women of reproductive age. Early diagnosis and management are important for the preservation of ovarian function. In cases of ovarian torsion, an ischemia/reperfusion (IR) injury may develop due to the release of free radicals and reactive oxygen species (ROS) during the detorsion process. ${ }^{1}$ Oxidative trauma occurs in a cell when the concentration of generated ROS exceeds that cell's antioxidant capability. ${ }^{2,3}$ Various antioxidants have been used for the prevention of oxidative injury and inflammation in ovaries subject to IR injury. ${ }^{4}$ Malondialdehyde (MDA) is the basic product of lipid peroxidation and is used to determine oxidative stress levels. ${ }^{5}$ It is well known that glutathione (GSH) is one of the most important indicators of the body's antioxidant capacity. ${ }^{1}$ Lycopene, a type of carotenoid, has antioxidant and chemopreventive properties. ${ }^{3}$ In our study, rats with ovarian torsion-detorsion damage were evaluated for the effects of lycopene. Moreover, the study evaluated the degree of ovarian tissue damage by histopathological examination and biochemically assessed the levels of MDA, GSH, myeloperoxidase (MPO), and superoxide dismutase (SOD) enzyme activity.

\section{Materials and Methods}

Approval from the Atatürk University Ethical Committee was obtained before the study (ethical approval date: 06.28.2018/07). The study was performed in the Animal Laboratory and Experimental Research Center of Atatürk University in July 2018. Animals were treated in accordance with the Guide for the Care and Use of Laboratory Animals (8th Edition, National Academies Press). In this study, 24 healthy, adult, nonpregnant, female Wistar Albino rats weighing between 228 and $272 \mathrm{~g}$ were used. The animals were fed and kept in a cage at a constant room temperature and humidity under standard 12-hour light and dark laboratory conditions. All experimental processes were performed when the rats were in the estrous phase. The rats were randomly divided into four groups of six animals: sham operation (group 1) $1 \mathrm{~mL}$ of corn oil was applied by gavage method; IR group 2: IR + $100 \mathrm{mg} / \mathrm{kg}$ lycopene, group 3: IR + $200 \mathrm{mg} / \mathrm{kg}$ lycopene, and group 4: lycopene 10\% FS (Redivivo, DSM Nutritional Products). Lycopene was suspended in corn oil and administered by gavage at doses of 100 and $200 \mathrm{mg}$ per $\mathrm{kg}$ half an hour before the end of ischemia ( 2.5 hours of ischemia). ${ }^{6}$

\section{Surgical Protocol}

All procedures were performed under general anesthesia and sterile conditions. Each rat was intramuscularly injected with $45 \mathrm{mg} / \mathrm{kg}$ of ketamine hydrochloride (Ketalar) and $5 \mathrm{mg} / \mathrm{kg}$ of xylazine hydrochloride (Rompun, Bayer) for general anesthesia. The skin of the abdomen was shaved and cleaned with $10 \%$ povidone iodine. The lower abdomen was opened with a 2-cm midline incision, and the bilateral ovary and adnexa were exposed. The uterine horn and ovaries were specified. Vascular clamps were placed just beneath the ovaries and over the uterine horns. The incisions were closed with $4-0$ silk sutures. The IR procedures were performed by one identical person using the same technique. The adnexa were rotated 720 degrees clockwise and fixed to the abdominal wall for 3 hours with a 5-0 polydioxanone suture. In the detorsion groups, the abdomen was reopened, and after the removal of the fixation sutures, the ovaries were brought to their former positions with detorsion. To protect the rats from hypothermia, the operating table was heated with a lamp from above and a heater from below. During the waiting period, the incision line on the abdominal region was closed with a 3-0 silk suture. In the sham group, a laparotomy was performed and the incision was closed with a 3-0 nylon suture. A relaparotomy was performed after a 3-hour period of all procedures in all groups, a bilateral oophorectomy was performed, and the animals were sacrificed after their blood was taken. In all groups, ovarian tissue was kept in $10 \%$ formaldehyde for histopathological evaluation and biochemical analyses.

\section{Biochemical Assay}

For the biochemical analyses, the ovarian tissues were dissected out and frozen immediately at $-80^{\circ} \mathrm{C}$. Then, $0.1 \mathrm{~g}$ of the tissue was homogenized with $900 \mu \mathrm{L}$ of ice-cold phosphate-buffered saline at $\mathrm{pH} 7.4(10 \% \mathrm{w} / \mathrm{v})$ and centrifuged at $4,000 \mathrm{rpm}$ for 15 minutes at $4^{\circ} \mathrm{C}$. The supernatants were stored at $-80^{\circ} \mathrm{C}$ to analyze the SOD, MPO, MDA, and GSH levels. The protein content of the supernatants was determined by the Bradford method using bovine serum albumin as standard. ${ }^{7}$ The MDA levels, an index of lipid peroxidation, were determined through thiobarbituric acid reaction using the method described by Ohkawa et al. ${ }^{8}$ The product was evaluated spectrophotometrically at $532 \mathrm{~nm}$, and the results are expressed as $\mathrm{nmol} / \mathrm{mg}$ protein. MPO activity in the supernatant was measured with the method described by Bradley et al. ${ }^{9}$ Then, $0.1 \mathrm{~mL}$ of the supernatant was mixed with $2.9 \mathrm{~mL}$ of $50-\mathrm{mM}$ phosphate buffer, $\mathrm{pH}$ 6.0, containing $0.167 \mathrm{mg} / \mathrm{mL}$ of O-dianisidine dihydrochloride and $0.0005 \%$ hydrogen peroxide. The change in absorbance at $460 \mathrm{~nm}$ was assayed spectrophotometrically. MPO enzyme activity was recorded as $\mathrm{U} / \mathrm{mg}$ protein in the sample. The GSH and SOD levels in the supernatants were measured with ELISA (enzyme-linked immunosorbent assay) methods using commercial kits (Cayman, cat no: 703002, and Cayman, cat no: 706002, respectively).

\section{Histopathological Examination}

The ovaries were fixed in $10 \%$ formaldehyde for 72 hours, dehydrated in a graded alcohol series, embedded in paraffin wax, and sectioned using a Leica RM2125RT microtome (Leica Microsystems). Five-mm-thick sections were used in this study for histopathological examinations and evaluations. After placing the 5-mm-thick sections of tissue onto slides and after deparaffinization and rehydration, one section from each rat was stained with hematoxylin-eosin. All sections were examined and photographed using a light photomicroscope (Olympus BX51 light microscope). Tissue damage was histopathologically assessed for hemorrhage, vascular congestion, and polymorphonuclear leukocyte 
(PMNL) infiltration. At least five microscopic regions were examined to score the specimens semiquantitatively. Each sample was scored for each criterion using a scale ranging from 0 to 3 ( 0 , none; 1 , mild; 2 , moderate; 3 , severe). Total scores were calculated from these parameters. One pathologist examined all the ovarian sections in a blinded fashion.

\section{Statistical Analysis}

The data regarding the biochemical SOD, GSH, MDA, and MPO levels were subjected to a one-way analysis of variance using the IBM SPSS Statistics Version 20.0 (IBM Corp.). Differences among the groups were determined using the Duncan multiple comparison test and were considered to be significant when the $p$-values were less than 0.05 . All the results were expressed as a mean standard deviation of the mean.

\section{Results}

\section{Biochemical Results of Ovary Tissues}

In this study, the antioxidant (SOD and GSH) and oxidant (MDA and MPO) parameters in the ovarian IR injury model in rats among different treatment groups are presented in

- Fig. 1. The MDA levels and MPO activity were significantly decreased, whereas the GSH levels and SOD activities were significantly increased in the ovarian tissues in the IR + lycopene groups compared with the IR group $(p<0.05)$. The SOD activity and GSH levels were found to be lower and the MDA levels and MPO activities were found to be higher in the IR group when compared with the IR + $100 \mathrm{mg}$ and IR +200 mg groups. Lycopene administration was found to ameliorate SOD activity and GSH levels in a dose-dependent manner. Furthermore, there were significant differences between the IR $+100 \mathrm{mg}$ and IR $+200 \mathrm{mg}$ groups for all antioxidant and oxidant parameters ( - Fig. 1$)(p<0.05)$. The SOD activity and GSH levels were found to be lower in the IR $+100 \mathrm{mg}$ lycopene group as compared with the IR + $200 \mathrm{mg}$ lycopene group. The MDA levels and MPO activities were found to be higher in the IR + $100 \mathrm{mg}$ lycopene group as compared with the IR + $200 \mathrm{mg}$ lycopene group. These results demonstrate an inverse correlation between lycopene levels and oxidative stress, supporting the hypothesis that lycopene supplementation could ameliorate oxidative stress in the rat ovary. Thus, in this study, the lowest SOD and GSH levels were observed in group 2. However, lycopene given before the reperfusion period increased SOD activity. The most dramatic increase occurred in group 4, which received the $200 \mathrm{mg} / \mathrm{kg}$ dose. The $200 \mathrm{mg} / \mathrm{kg}$ dose of lycopene was more effective than the $100 \mathrm{mg} / \mathrm{kg}$ dose.

\section{Histopathological Results}

All rats were evaluated for tissue damage by assessing parameters such as congestion, PMNL infiltration, and hemorrhage. The histopathological scores for all four groups are listed. The ovarian histopathological changes for the IR, IR + lycopene $100 \mathrm{mg}$, and IR + lycopene $200 \mathrm{mg}$ groups are shown in - Fig. 2. The histopathological evaluation showed that tissue damage significantly increased in the IR group compared with the lycopene treatment groups. The tissue damage in the lycopene treatment groups significantly decreased compared with the IR group. Cellular improvements on these histopathological parameters as a result of lycopene can be a reflection of its antioxidant effect. Vascular congestion, hemorrhage, and PMNL infiltration were significantly higher in the IR group than in the sham group. Congestion, hemorrhage, and PMNL infiltration regressed to the degrees of mild and medium from severe in the IR group, which was given the lycopene treatment. There were significant differences in tissue damage between the lycopene $100 \mathrm{mg}$ and lycopene $200 \mathrm{mg}$ groups. The sham group (group 1) had a normal histological architecture with minimal congestion, including the cortex and medulla. The cortex consists of ovarian follicles and corpus luteum, whereas the medulla consists of vascular
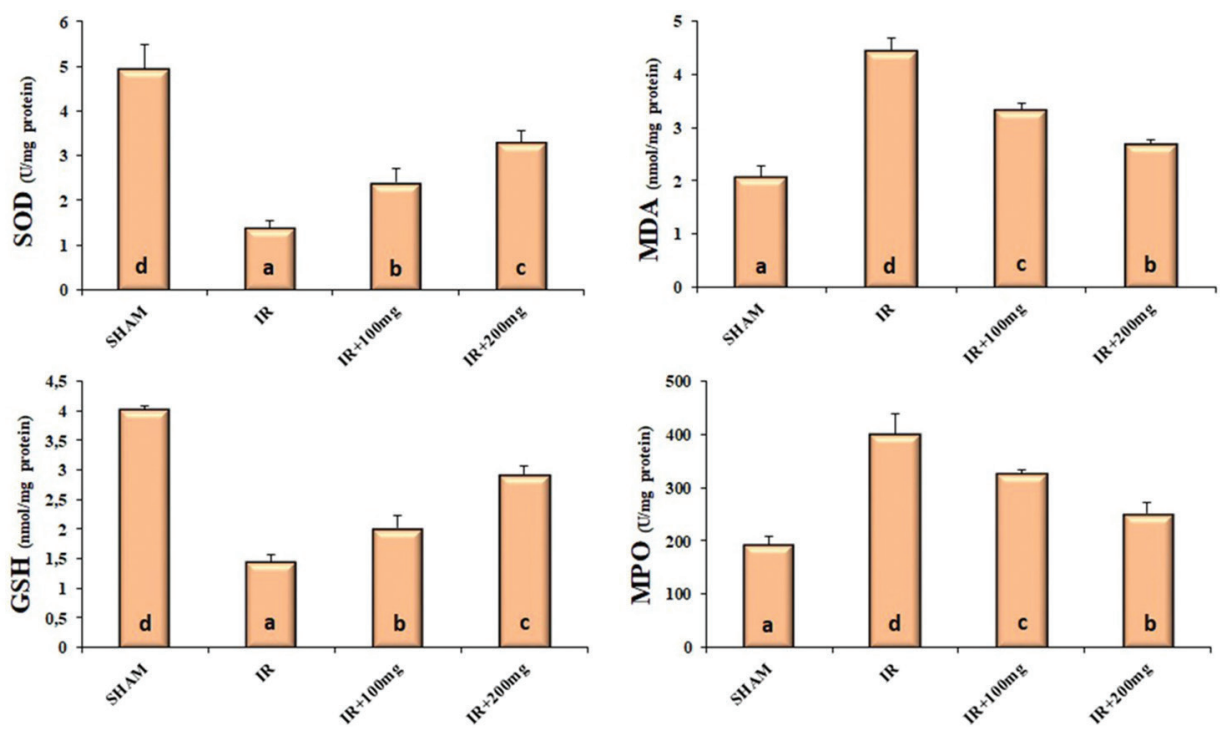

Fig. 1 Effects of lycopene treatments on SOD and MPO activities, and GSH and MDA levels in rat ovaries. Means in the same column by the same letter are not significantly different to the Duncan test $(p<0.05)$. Results are presented as means \pm standard deviation. GSH, glutathione; IR, ischemia/reperfusion; MDA, malondialdehyde; MPO, myeloperoxidase; SHAM, sham-operated; SOD, superoxide dismutase. 


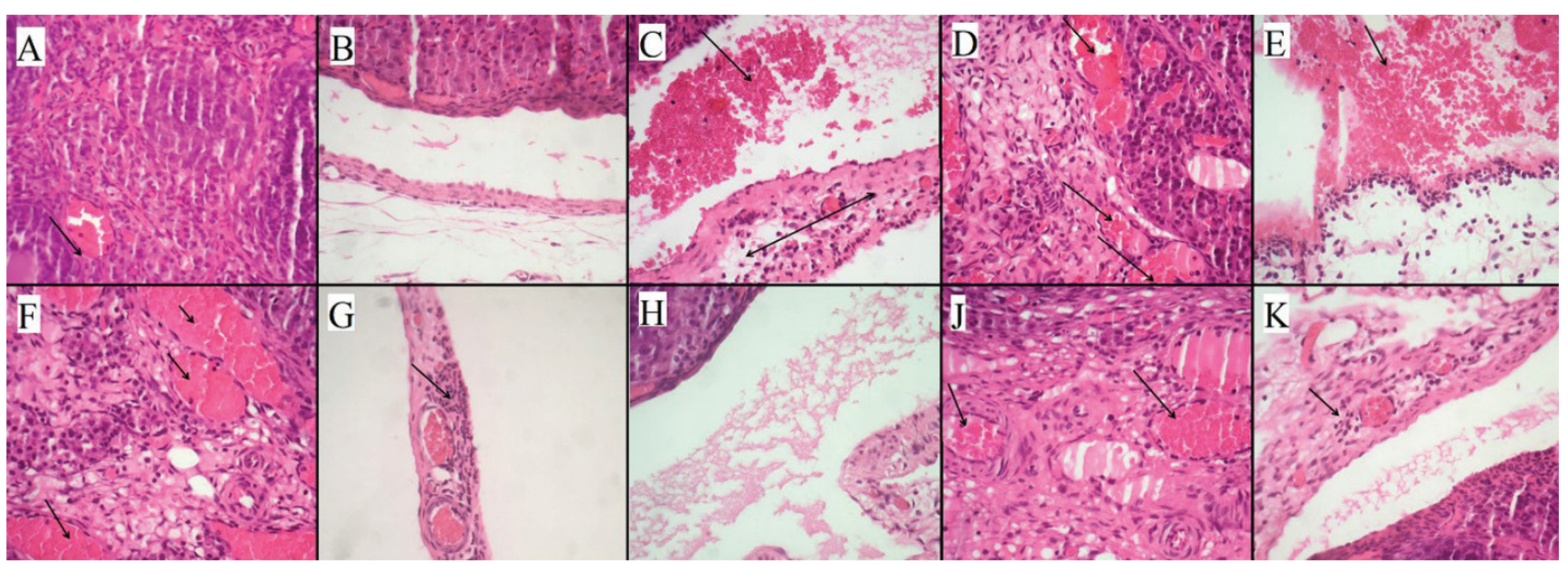

Fig. 2 Effects of lycopene treatments on light micrographs in rats' ovaries (hematoxylin-eosin (H\&E) staining). (A) Sham group (group 1) with minimal congestion, x400, H\&E. (B) Sham group, (group 1) with no inflammation and hemorrhage, $x 400$, H\&E. (C) Ischemia/reperfusion (IR) group (group 2) with hemorrhage (unidirectional arrow) and inflammation (bidirectional arrow), x400, H\&E. (D) IR group (group 2) with congestion (arrow) x400, H\&E. (E) IR+ lycopene $100 \mathrm{mg} / \mathrm{kg}$ (group 3) with focal hemorrhage, x400, H\&E. (F) IR+ lycopene $100 \mathrm{mg} / \mathrm{kg}(\mathrm{group} 3)$ with congestion, $x 400, H \& E$. (G) IR+ lycopene $100 \mathrm{mg} / \mathrm{kg}$ (group 3) with inflammation (arrow), x400, H\&E. (H) IR+ lycopene $200 \mathrm{mg} / \mathrm{kg}$ (group 4) with hemorrhage not detected, $x 400$, H\&E. (J) IR + lycopene $200 \mathrm{mg} / \mathrm{kg}$ (group 4) with minimal congestion, x400, H\&E. (K) IR+ lycopene $200 \mathrm{mg} / \mathrm{kg}$ (group 4) with minimal inflammation, $\mathrm{x} 400, \mathrm{H} \& \mathrm{E}$.

structures and connective tissue. The tunica albuginea that surrounds the ovary had a normal appearance ( - Fig. 2 A,B $)$. In the ovarian torsion IR group (group 2), intensive neutrophil infiltration was observed in the peripheral connective tissue, and hemorrhage was observed between this tissue and the ovarian tissue ( - Fig. 2C). Significant congestion was observed in the vascular structures in the medulla (-Fig. 2D). The ovarian torsion $+100 \mathrm{mg} / \mathrm{kg}$ lycopene group (group 3 ) had focal hemorrhage compared with the IR group (-Fig. 2E). In the IR + lycopene $100 \mathrm{mg}$ group, congestion and inflammatory cell density were lower than in the IR group ( - Fig. 2F,G). Hemorrhage was not observed in the ovarian torsion $+200 \mathrm{mg} / \mathrm{kg}$ lycopene group (group 4) ( - Fig. $\mathbf{2 H}$ ). The congestion and infiltration of infective cells were minimal ( - Fig. 2J,K) in all groups.

\section{Discussion}

Ovarian torsion frequently occurs in women during the premenarchal or reproductive years. For that reason, early and accurate diagnosis is very important for a woman's reproductive health. ${ }^{10}$ Ovarian torsion must be diagnosed and treated to prevent potential necrosis leading to infertility. ${ }^{11}$ Detorsion might be considered to restore the ovarian blood flow. However, reperfusion might lead to more serious injury, ${ }^{12}$ known as an IR injury. ${ }^{13}$ In oxidative stress, there is an imbalance between the production and elimination of ROS. ${ }^{3}$ The IR condition oxidizes cellular membrane lipids and leads to the formation of toxic products such as MDA..$^{14}$ On the other hand, cellular protection against oxidative damage is provided by antioxidant enzymes and nonenzymatic compounds such as GSH or SOD. ${ }^{4,11}$ MPO, a major component of neutrophil azurophilic granules, is often released from stimulated PMNLs at inflammation sites and is involved in the generation of ROS. ${ }^{15}$ MDA is the end product of lipid peroxidation, which induces ischemic injury, whereas GSH is the most significant cellular antioxidant compound. ${ }^{16}$ It defends cells against oxidant damage by entering into reactions with GSH and free radicals. ${ }^{11}$ SOD and MPO are antioxidant enzyme components of the defense mechanism against the activities of oxidative substances. Increased levels of these enzymes protect the tissue during ovarian IR damage.,17,18 After ovarian detorsion, to protect the ovarian reserve against IR damage, prophylactic measures are necessary. ${ }^{19}$ Therefore, several studies have focused on pharmaceutical agents with antioxidant effects to prevent ovarian IR damage in animal models. ${ }^{13,3,18}$

The ischemia that develops due to torsion has been reported to increase the blood levels of lipid peroxidation products. ${ }^{20}$ It is known that antioxidant treatment helps prevent tissue damage related to increases in oxidant production. Antioxidant agents reduce oxidized biomolecules and repair existing oxidative injury. ${ }^{21}$ Therefore, to prevent ischemic injury in ovarian torsion, many prophylactic agents such as erdosteine, selenium, erythropoietin, vardenafil, vitamin C, and curcumin have been used before and after ischemia. ${ }^{22,23}$

Cellular and molecular studies have revealed lycopene, which not only inhibits lipid peroxidation but also quenches $\mathrm{ROS},{ }^{24}$ to be one of the strongest antioxidants. ${ }^{25}$ It has been commonly used as an antioxidant agent in traditional medicine and has been reported to have protective effects for the treatment of cardiovascular diseases, neurotoxicity, hepatic injury, and nephrotoxicity. ${ }^{26}$

Acetaminophen overdoses are causes of hepatic necrosis and acute liver failure. ${ }^{27}$ It has been reported that lycopene defends against acetaminophen-induced liver injury in mice by increasing antioxidant substances including GSH. ${ }^{28}$ Carbon tetrachloride $\left(\mathrm{CCl}_{4}\right)$ causes liver injury in experimental studies. ${ }^{29}$ In the study by Pinto et al, it was shown that lycopene acted as a therapeutic agent against $\mathrm{CCl}_{4}$-induced acute liver damage in experimental animals. A significant 
increase was observed in the GSH concentration and SOD activity in lycopene-treated groups. ${ }^{30}$ Previous studies have reported that gentamicin nephrotoxicity is related to oxidative stress and hydroxyl radicals. ${ }^{31}$ Studies on the prevention of gentamicin toxicity reported that treatment with lycopene markedly increased antioxidant levels and reduced the urea and creatinine levels in rats given gentamicin. ${ }^{32}$ Cyclosporine $\mathrm{A}$ is used as an immunosuppressive drug after transplantation, but it has nephrotoxicity side effects. ${ }^{33}$ It was stated that giving lycopene to rats caused a decrease in the urea and creatinine serum concentrations, an increase in SOD, and the suppression of MDA. ${ }^{34}$ A study by Liu et al reported that lycopene ameliorates ovarian aging in chickens. The ovarian tissues of young and old hens were treated with lycopene to verify its protective effects. Treatment with lycopene reported a decrease in the MDA content and ROS levels in both young and old ovarian tissues. ${ }^{35}$

Many studies have shown that MDA levels increased related IR injuries. ${ }^{36}$ It has been stated that this increase is a clear sign of an IR injury. ${ }^{37}$ In our study, the histopathological and biochemical evaluations showed that 100 or $200 \mathrm{mg}$ doses of lycopene were effective in reducing ovarian tissue damage. Furthermore, when compared with the sham operated group, the increase in the MDA level in the IR group was significant. On the other hand, the IR process caused a significant decrease in the GSH level in the ovarian tissue of rats compared with the sham group. Lycopene significantly improved the GSH level in the lycopene treatment groups when compared with the IR group. The SOD activity was suppressed in the IR group compared with the sham group. But a significant increase was found in the lycopene treatment groups compared with the IR group. On the contrary, MPO activity was significantly increased in the IR group when compared with the sham group, and when lycopene was supplied, MPO activity significantly decreased. Consistent with our results, Tok et al reported that the levels of oxidant indicators such as MDA and MPO increased and the levels of antioxidant indicators such as GSH decreased in the IR group. ${ }^{38}$

On the contrary, Sayar at al showed that a significant rise in the SOD enzyme activity was detected in the IR group, whereas a significant reduction in the levels of this marker was observed when antioxidant material was applied to an IR injury. ${ }^{39}$

Histopathologically, dilated congested blood vessels, inflammation, and severe hemorrhage in ovarian tissue with the oxidative trauma caused by IR were also observed in this study. Confirming our results, similar histopathological changes in rat ovaries related to IR injury were reported in previous experimental studies. ${ }^{1,40}$

An increase in antioxidant enzyme levels is a reflection of increased oxidative stress. In addition, it was detected that enzyme levels decreased in the rats induced with an IR when administered lycopene. These results are also important in terms of the antioxidant effects of lycopene that might be a result of toxic agents resulting from its administration. In our study, the histopathological and biochemical evaluations showed that lycopene was effective in reducing ovarian tissue damage. It was observed that the dose-dependent activity increased.

In conclusion, the results of this study showed that lycopene has protective effects on oxidative stress induced by ischemia in ovarian torsion and increases the dose-dependent effect. However, further clinical studies are required to reach accurate results.

\section{Author Contribution}

P. T. Y. contributed to data collection/management and protocol/project development. H. U. contributed to manuscript writing/editing and data analysis. B. G. contributed to data analysis. E. P. contributed to protocol/project development. S. A. contributed to protocol/project development. Y E. T. contributed to data collection/management. Z. H. contributed to data analysis and protocol/ project development.

\section{Funding}

The authors declared that this study received no financial support.

\section{Ethics Committee Approval}

Ethics committee approval was received for this study from the ethics committee of Ataturk University. Animals were treated in accordance with the Guide for the Care and Use of Laboratory Animals (8th Edition, National Academies Press).

\section{Conflict of Interest}

No conflict of interest was declared by the authors.

\section{References}

1 Bayir Y, Cadirci E, Polat B, et al. Aliskiren - a promising strategy for ovarian ischemia/reperfusion injury protection in rats via RAAS. Gynecol Endocrinol 2016;32(8):675-683

2 Oral A, Halici Z, Bayir Y, et al. Effects of oral zinc administration on long-term ipsilateral and contralateral testes damage after experimental testis ischaemia-reperfusion. Andrologia 2017;49(6):e12673

3 Sahin K, Yenice E, Tuzcu M, et al. Lycopene protects against spontaneous ovarian cancer formation in laying hens. J Cancer Prev 2018;23(1):25-36

4 Oral A, Odabasoglu F, Halici Z, et al. Protective effects of montelukast on ischemia-reperfusion injury in rat ovaries subjected to torsion and detorsion: biochemical and histopathologic evaluation. Fertil Steril 2011;95(4):1360-1366

5 Yigiter M, Halici Z, Odabasoglu F, et al. Growth hormone reduces tissue damage in rat ovaries subjected to torsion and detorsion: biochemical and histopathologic evaluation. Eur J Obstet Gynecol Reprod Biol 2011;157(1):94-100

6 Cakir Gungor AN, Gencer M, Karaca T, et al. The effect of hesperetin on ischemia-reperfusion injury in rat ovary. Arch Gynecol Obstet 2014;290(4):763-769

7 Bradford MM. A rapid and sensitive method for the quantitation of microgram quantities of protein utilizing the principle of protein-dye binding. Anal Biochem 1976;72:248-254

8 Ohkawa H, Ohishi N, Yagi K. Assay for lipid peroxides in animal tissues by thiobarbituric acid reaction. Anal Biochem 1979;95(2):351-358

9 Bradley PP, Priebat DA, Christensen RD, Rothstein G. Measurement of cutaneous inflammation: estimation of 
neutrophil content with an enzyme marker. J Invest Dermatol 1982;78(3):206-209

10 Eser A, Hizli D, Haltas H, et al. Effects of curcumin on ovarian ischemia-reperfusion injury in a rat model. Biomed Rep 2015;3(6):807-813

11 Halici Z, Karaca M, Keles ON, et al. Protective effects of amlodipine on ischemia-reperfusion injury of rat ovary: biochemical and histopathologic evaluation. Fertil Steril 2008;90(6):2408-2415

12 Zimmerman BJ, Granger DN. Reperfusion injury. Surg Clin North Am 1992;72(1):65-83

13 Carden DL, Granger DN. Pathophysiology of ischaemia-reperfusion injury. J Pathol 2000;190(3):255-266

14 Un H, Bayir Y, Halici Z, et al. The effects of RAAS Inhibition in rate limiting step by Aliskiren on testicular torsion injury in rats. J Urol 2015;194(3):828-833

15 Ece A, Kelekçi S, Hekimoğlu A, et al. Neutrophil activation, protein oxidation and ceruloplasmin levels in children with Henoch-Schönlein purpura. Pediatr Nephrol 2007;22(8):1151-1157

16 Kaya C, Karabulut R, Turkyilmaz Z, et al. Lycopene has reduced renal damage histopathologically and biochemically in experimental renal ischemia-reperfusion injury. Ren Fail 2015;37(8):1390-1395

17 Dogan C, Halici Z, Topcu A, et al. Effects of amlodipine on ischaemia/reperfusion injury in the rat testis. Andrologia 2016;48(4):441-452

18 Cadirci E, Oral A, Odabasoglu F, et al. Atorvastatin reduces tissue damage in rat ovaries subjected to torsion and detorsion: biochemical and histopathologic evaluation. Naunyn Schmiedebergs Arch Pharmacol 2010;381(5):455-466

19 Halestrap AP, Clarke SJ, Javadov SA. Mitochondrial permeability transition pore opening during myocardial reperfusion-a target for cardioprotection. Cardiovasc Res 2004;61(3):372-385

20 Akgür FM, Kilinç K, Aktuğ T. Reperfusion injury after detorsion of unilateral testicular torsion. Urol Res 1993;21(6):395-399

21 Kisaoglu A, Borekci B, Yapca OE, Bilen H, Suleyman H. Tissue damage and oxidant/antioxidant balance. Eurasian J Med 2013;45(1):47-49

22 Sağsöz N, Kisa U, Apan A. Ischaemia-reperfusion injury of rat ovary and the effects of vitamin C, mannitol and verapamil. Hum Reprod 2002;17(11):2972-2976

23 Yildirim N, Simsek D, Kose S, et al. The protective effect of Gingko biloba in a rat model of ovarian ischemia/reperfusion injury: improvement in histological and biochemical parameters. Adv Clin Exp Med 2018;27(5):591-597

24 Kawata A, Murakami Y, Suzuki S, Fujisawa S. Anti-inflammatory activity of $\beta$-carotene, lycopene and tri-n-butylborane, a scavenger of reactive oxygen species. In Vivo 2018;32(2):255-264

25 Basu A, Imrhan V. Tomatoes versus lycopene in oxidative stress and carcinogenesis: conclusions from clinical trials. Eur J Clin Nutr 2007;61(3):295-303
26 Hedayati N, Naeini MB, Nezami A, et al. Protective effect of lycopene against chemical and natural toxins: a review. Biofactors 2019;45(1):5-23

27 Larson AM, Polson J, Fontana RJ, et al; Acute Liver Failure Study Group. Acetaminophen-induced acute liver failure: results of a United States multicenter, prospective study. Hepatology 2005;42(6):1364-1372

28 Bandeira ACB, da Silva RC, Rossoni JV, et al. Lycopene pretreatment improves hepatotoxicity induced by acetaminophen in C57BL/6 mice. Bioorg Med Chem 2017;25(3):1057-1065

29 Brattin WJ, Glende EA Jr, Recknagel RO. Pathological mechanisms in carbon tetrachloride hepatotoxicity. J Free Radic Biol Med 1985;1(1):27-38

30 Pinto C, Rodriguez-Galdon B, Cestero JJ, Macias P. Hepatoprotective effects of lycopene against carbon tetrachloride-induced acute liver injury in rats. J Funct Foods 2013;5(4):1601-1610

31 Walker PD, Barri Y, Shah SV. Oxidant mechanisms in gentamicin nephrotoxicity. Ren Fail 1999;21(3-4):433-442

32 Karahan I, Ateşşahin A, Yilmaz S, Ceribaşi AO, Sakin F. Protective effect of lycopene on gentamicin-induced oxidative stress and nephrotoxicity in rats. Toxicology 2005;215(3):198-204

33 Cohen DJ, Loertscher R, Rubin MF, Tilney NL, Carpenter CB, Strom TB. Cyclosporine: a new immunosuppressive agent for organ transplantation. Ann Intern Med 1984;101(5):667-682

34 Ateşşahin A, Ceribaşi AO, Yilmaz S. Lycopene, a carotenoid, attenuates cyclosporine-induced renal dysfunction and oxidative stress in rats. Basic Clin Pharmacol Toxicol 2007;100(6):372-376

35 Liu X, Lin X, Zhang S, et al. Lycopene ameliorates oxidative stress in the aging chicken ovary via activation of Nrf2/HO-1 pathway. Aging (Albany NY) 2018;10(8):2016-2036

36 Celik M, Aksoy AN, Aksoy H, Aksoy Y, Halici Z. Sildenafil reduces ischemia-reperfusion injury in rat ovary: biochemical and histopathological evaluation. Gynecol Obstet Invest 2014;78(3):162-167

37 Erten SF, Kocak A, Ozdemir I, Aydemir S, Colak A, Reeder BS. Protective effect of melatonin on experimental spinal cord ischemia. Spinal Cord 2003;41(10):533-538

38 Tok A, Sener E, Albayrak A, et al. Effect of mirtazapine on oxidative stress created in rat kidneys by ischemia-reperfusion. Ren Fail 2012;34(1):103-110

39 Sayar I, Bicer S, Gursul C, Gürbüzel M, Peker K, Işik A. Protective effects of ellagic acid and ozone on rat ovaries with an ischemia/ reperfusion injury. J Obstet Gynaecol Res 2016;42(1):52-58

40 Yapca OE, Kumbasar S, Salman S, et al. Controlled reperfusion for different durations in the treatment of ischemia-reperfusion injury of the rat ovary: evaluation of biochemical features, molecular gene expression, and histopathology. Can J Physiol Pharmacol 2015;93(4):269-274 